\title{
Surgical Breast Specialists 2.0: Rethinking the Future of Breast Fellowship Training
}

\author{
Peter D. Beitsch, MD, FACS ${ }^{1}$ and Pat Whitworth, MD, FACS ${ }^{2}$ \\ ${ }^{1}$ Dallas Breast Center, Dallas, TX; ${ }^{2}$ Nashville Breast Center, Nashville, Tennessee
}

The timely wake-up call by Kurtzman echoes the first part of the presidential address delivered at the American Society of Breast Surgeons (ASBrS) in 2014. That address was delivered by one of us (Beitsch), and fortunately there was a second part outlining a bright future and a path forward. ${ }^{1}$ The inescapable fact that change is coming, as emphasized by Kurtzman, means that to lead effectively, breast surgeons must adapt and embrace the advances that are underway. The ASBrS was established by breast surgeons who recognized early on that ultrasound and other imaging technologies would ultimately guide treatment as well as diagnostic biopsy. Certification in breast ultrasound and stereotactic-guided procedures was one of the earliest and is one of the highest valued ASBrS programs. It has long been the position of the ASBrS that breast surgeons are most effective when they use these skills routinely in practice.

There is no doubt that all of general surgery has been dramatically changing for many years. For the past several decades, laparoscopic procedures, endovascular procedures, bariatric operations (open and laparoscopic), complex hernias, endoscopic treatments, and on and on have changed - and continue to change-general surgery training. This is also true for surgical breast treatments, the largest being the drastic (and appropriate) drop in open excisional biopsies, mastectomies, and axillary dissections. Further stressing general surgery training was the advent of breast fellowships and the competition for the care of patients with benign and malignant breast problems. But the focus of this editorial is specifically the future of

(C) Society of Surgical Oncology 2016

First Received: 3 June 2016;

Published Online: 22 June 2016

P. D. Beitsch, MD, FACS

e-mail: beitsch@aol.com surgical breast specialists. We have a somewhat different (and more optimistic) view of the potential bright future for breast fellows if surgical educators can move away from the traditional breast surgical training of breast fellows to a broader and comprehensive management of breast-related issues as we move toward the era when we will consider any patient who develops advanced breast cancer as a failure of health care. BRCA mutations carriers who develop advanced breast cancer are a current obvious example of our failure to deliver the testing and care we have within our grasp.

The advances outlined below will not occur overnight, and may not occur at all, but in our view, this is the way the future is headed, although the pace is yet to be determined.

\section{BENIGN BREAST DISEASE}

Successful breast specialists will be more than cancer surgeons. A major component of breast specialist care will be in the benign realm. It is absolutely imperative that breast specialist training include taking care of breast pain, infections including abscesses, nipple discharge, skin changes (usually benign), benign lumps including benign tumors, and imaging abnormalities that are not ultimately found to be a cancer. This means training will include a healthy experience in the breast clinic and strong, skilled mentors with all of the skills under discussion here. Programs without such mentors will collaborate with leading community breast surgeons who have these skills.

\section{RISK ASSESSMENT}

Some women have a deleterious genetic mutation and are at higher than average risk of developing a breast cancer; by definition, some women must be at a lower risk such that the average is maintained. Deciding a woman's risk of developing a breast cancer will have to move 
beyond patient age (starting mammography at 40 or 45) and sex (women need screening and men don't). Breast specialists must be facile in using the risk models that we currently have (Gail, Claus, Tyrer-Cuzick, BRACAPRO, etc.) and staying current as they evolve. ${ }^{2}$ Risk assessment is the cornerstone of screening as we move from general population screening to more personalized screening and diagnosis based on individualized risk assessment. By definition, this first involves assessing risk. Breast specialists who haven't should seize risk assessment immediately. They are probably best at directing the appropriate surveillance and imaging needed and then dealing with the results.

\section{ULTRASOUND AND IMAGE-GUIDED BIOPSY}

Many surgical specialties use ultrasound to aid in diagnosis (including biopsy) and treatment, including endocrine, urology, trauma, obstetrics, gynecology, and hepato-pancreatico-biliary. Ultrasound is not and should not be restricted to the radiologist. Ultrasound aids in evaluating breast pain, lumps, discharge, and adenopathy, and it is critical to guide needles for biopsy (fine needle aspiration, core biopsy, and percutaneous excisions). Furthermore, it is a skill that can be used to localize breast lesions (or most clips) in the operating room, including cancers and can lead to reduced positive margins. Along with ultrasound-guided procedures, breast specialists should be able to do stereotactic procedures (core biopsies and minimally invasive excisions). The often repeated excuse, "We have excellent radiologists just down the hall ..." will lead to the sad outcome outlined in Kurtzman's excellent call to action. If breast specialists do risk assessment and order the targeted imaging, they will be doing the image-guided procedures.

\section{BLOOD-BASED DIAGNOSIS BASED ON INDIVIDUALIZED RISK}

There are already several companies developing bloodbased tests with high negative predictive value (ie similar to BIRADS 3 imaging with $<2 \%$ chance of cancer in an imaged finding) that will be used to screen at-risk women. A positive test will lead to diagnostic imaging and either image-guided tissue biopsy or blood-based liquid biopsy. The subsequently discovered breast cancer will undergo comprehensive genomic analysis.

\section{TARGETED TREATMENT}

If breast specialists are assessing risk, ordering imaging, performing biopsies (image guided and drawing blood), then they will be guiding the targeted treatment plan for the breast cancer patient. This will include two large groups of patients (although there will be overlap): first, a substantial group of tumors that have little ability to metastasize systemically (usually luminal A [estrogen receptor positive, progesterone receptor positive, HER-2 negative]), and second, a group of tumors that are more likely to be systemic by the time they are diagnosed (luminal B, basal, and HER-2 positive). For locoregional cancers, the breast specialist will excise the primary lesion (often minimally invasive excision with image guidance) and (for a little longer) remove the sentinel nodes in most cases, since this affects the targeted radiation that will follow. The breast specialist will then administer and monitor hormone therapy. For systemic-oriented cancers, breast specialists will direct the patient to an advanced breast medical oncologist for intravenous and/or oral targeted biologics and/or chemotherapy, depending on molecular profile. There is already a model for dividing the systemic therapy into basic (surgeon) and complex (medical oncology): that of prostate cancer, another complex cancer with local and systemic varieties. Urologists routinely provide hormone therapy, including injections and oral medications. If the disease fails to respond to initial therapy, or if the patient starts with a complex systemic cancer, advanced breast medical oncologists will prescribe systemic treatment. Breast specialists must move into this space not only to remain relevant but also to address the coming shortage of medical oncologists.

\section{GENETIC TESTING AND PROPHYLACTIC MASTECTOMIES}

There is currently a revolution occurring in genetic testing, including many new companies offering increasingly broad cancer panels. (In addition are panels being offered for other disease-related genes.) This is both an incredible opportunity to identify people at risk of geneticrelated cancers (despite $>20$ years of $B R C A 1 / 2$ testing, $<10$ $\%$ of all $B R C A$ mutation carriers have been identified, and far less for other cancer-related gene carriers) and a conundrum because of the dearth of genetic counselors available. ${ }^{3}$ Genetic counselors will help lead screening and testing programs, as opposed to their current misallocation as gatekeepers for genetic testing. Breast specialists should take the lead in educating themselves and their fellows about these genes and the consequences of deleterious mutations. Soon a deadly breast cancer that becomes clinically apparent in a patient with a deleterious mutation will be considered a failure of medicine because most of these cancers can be prevented or detected while in a curable state. Large groups of patients at moderate to high 
to very high risk of developing breast (and other) cancers will be identified and will need to be monitored and managed. Part of this management for some patients (in a shared decision-making environment) will be prophylactic mastectomies (often nipple sparing) with reconstruction. Surgical breast specialists will be performing these extirpative operations if they are the ones ordering and counseling the patients. Initially these patients will be identified by risk models, but soon, within 1 to 5 years, they will be getting comprehensive testing, perhaps even whole genome sequencing, on their own, or in many cases via their primary care providers. Breast specialists should be ready to manage these people not only for their breast cancer risk but for other associated neoplasms, including pancreatic cancer, melanoma, uterine and ovarian cancer, and colon cancer. Surgical breast fellows should become facile at nipple-sparing prophylactic and therapeutic mastectomies as well as relatively simple reconstructions, such as expanders/implants. The sheer volume of these cases in the future will necessitate simple reconstruction falling to the surgical breast specialist, saving the complex reconstructions to plastic surgeons.

\section{RECURRENCE MONITORING AND SALVAGE SURGERY}

Despite our best efforts, some patients will experience recurrence locally, regionally, or systemically. Breast specialists should be the ones monitoring for these recurrences with blood tests (circulating DNA), imaging, and physical examinations. Who is better equipped to examine and diagnose recurrence than breast specialists? Many, perhaps most, recurrences will be handled with targeted systemic therapy, but there will be a need for surgery at times. These could include salvage mastectomies; axillary, cervical, and even internal mammary node dissections; and chest wall resections. Most of these will be difficult operations and will undoubtedly be done at high-volume centers, where, we hope, there will be surgical breast fellows in training.

\section{SURVIVORSHIP MONITORING AND TREATMENT}

Until we stop the development of breast cancer through environmental improvements and Crispr/cas9 repair of deleterious genes, there will be increasing numbers of breast cancer survivors with treatment-related problems that will need management. These include lymphedema, neuropathy/pain syndromes, and menopause symptoms. Lymphedema is best treated when there is minimal fluid accumulation, so early (even preclinical) detection is the key to preventing long-term sequelae. Surgical breast specialists (along with radiation oncologists) cause this problem, so we should be at the forefront of diagnosing and managing treatment. Similarly, locoregional treatment can lead to neuropathic pain syndromes that are not treated with narcotics. Unaddressed pain syndromes can be severe, unrelenting, and disabling, so early diagnosis and appropriate treatment is imperative. Last, our treatments (chemotherapy, hormone therapy, and even surgical removal) can cause menopause symptoms of varying severity. Our goal should be to care for the entire person, not just her breast cancer, and therefore we should try to restore a woman's life to as near its precancer state as we can. This may involve many different treatments for hot flashes (including antidepressants, complementary medicine, and even testosterone therapy), sexual dysfunction (including lubricants and topical estrogen), and bone mineral loss (including exercise, calcium/vitamin D, and bisphosphonates).

Surgical breast specialization has the potential for a long and prosperous period, but it will look quite different from the current breast cancer-oriented surgery. As such, breast fellowship coordinators should immediately begin reevaluating the curriculum.

DISCLOSURE The authors declare no conflict of interest.

\section{REFERENCES}

1. Beitsch PD. American Society of Breast Surgeons presidential address: breast cancer surgery is dead...or is it? Ann Surg Oncol. 2014;21:3166. doi:10.1245/s10434-014-3910-4.

2. CRA Health. Hughes risk apps. http://www.crahealth.com/.

3. Drohan B, Roche CA, Cusack JC Jr, Hughes KS. Hereditary breast and ovarian cancer and other hereditary syndromes: using technology to identify carriers. Ann Surg Oncol. 2012;19:1732-7. 